\title{
Visual Areas in Macaque Cortex Measured Using Functional Magnetic Resonance Imaging
}

\author{
Alyssa A. Brewer, ${ }^{1}$ William A. Press, ${ }^{2}$ Nikos K. Logothetis, ${ }^{3}$ and Brian A. Wandell ${ }^{1,2}$ \\ ${ }^{1}$ Neuroscience Program and 2Department of Psychology, Stanford University, Stanford, California 94305, and \\ 3Max-Planck Institut für Biologische Kybernetik, 72076 Tübingen, Germany
}

\begin{abstract}
We describe the first systematic functional magnetic resonance imaging (fMRI) measurements of visual field maps in macaque visual cortex. The boundaries of visual areas V1, V2, V3, V3A, V4, MT/V5, and TEO/V4A were identified using stimuli that create traveling waves of activity in retinotopically organized areas of the visual cortex. Furthermore, these stimuli were used to measure the dimensions of the representations of the central $11^{\circ}$ in V1-V3, quantitative visual field eccentricity functions for $\mathrm{V} 1-\mathrm{V} 3$ and $\mathrm{MT}$, and the distribution of foveal and peripheral signals within the occipital lobe. Within areas V1, V2, MT, and portions of $\mathrm{V} 4$, the $\mathrm{fMRI}$ signals were 5-10 times the noise level ( $3 \mathrm{~mm}^{3}$ volumes of interest). Signals were weaker but still significant in other cortical regions, including V3, V3A, and TEO.
\end{abstract}

There is good agreement between the fMRI maps and the visual area maps discovered using local anatomical and physiological measurements. The fMRI measurements allow one to obtain a broad view of the distribution of cortical signals, spanning multiple visual areas at a single point in time. The combination of scale and sensitivity demonstrated here create a good foundation for measuring how localized signals and lesions influence the responses and reorganization in widely separated cortical regions. The ability to measure human and macaque maps using the same technology will make it possible to define computational homologies between the two species.

Key words: visual cortex; visual areas; cortical magnification; fMRI; monkey; human; extrastriate cortex
Functional magnetic resonance imaging (fMRI) provides new perspectives on the organization of visual cortex in both human and monkey brains (Logothetis et al., 1999; Wandell, 1999; Tolias et al., 2001; Vanduffel et al., 2001; Van Essen et al., 2001; Sereno et al., 2002). At present levels of sensitivity, human fMRI is useful for visualizing activity of the whole brain with millimeter resolution (Engel et al., 1997). In conventional monkey fMRI experiments, resolution can be at the submillimeter level (Logothetis et al., 2001), and for smaller volumes of interest, the use of implanted radio frequency coils permits voxel sizes as small as $0.012 \mu \mathrm{l}(0.125 \times 0.125 \times 0.770$ $\mathrm{mm}^{3}$; Logothetis et al., 2002).

Using fMRI in a $4.7 \mathrm{~T}$ magnet and an anesthetized monkey, Logothetis et al. (1999) made preliminary measurements of the visual field organization in macaque. Here, by using the traveling wave methods developed in human (Engel et al., 1994; Sereno et al., 1995; DeYoe et al., 1996; Engel et al., 1997), we describe the first systematic fMRI measurements of visual field maps in macaque visual cortex.

The visual field maps clearly delineate several visual areas. In addition, the maps clarify the predominance of the foveal and peripheral signals within the ventral and dorsal streams, respectively (Morel and Bullier, 1990; Baizer et al., 1991). Although the fMRI signals from anterior visual cortex are weaker than those in posterior cortex, the signals are adequate

Received April 29, 2002; revised Aug. 20, 2002; accepted Sept. 13, 2002.

This work was supported by National Eye Institute Grant RO1 EY03164 and the Max-Planck Society. We thank Alex Wade, Robert Dougherty, Bill Newsome, and Semir Zeki for help.

Correspondence should be addressed to Alyssa A. Brewer, Wandell Laboratory, Room 490, Jordan Hall, Building 420, Stanford University, Stanford, CA 94305. E-mail: alyssa.brewer@stanford.edu.

Copyright (C) 2002 Society for Neuroscience $0270-6474 / 02 / 2210416-11 \$ 15.00 / 0$ to identify several anterior visual field maps. With continuing improvements in methods, fMRI data should help resolve some of the differences among visual cortex partitioning schemes (Gattass et al., 1988; Kaas and Lyon, 2001; Van Essen et al., 2001).

Finally, the measurements are very stable across scans, and once instrumentation and protocols are established, data spanning large regions of cortex are relatively easy to obtain. The combination of sensitivity and scale is a good foundation for measuring how localized signals and lesions influence responses and reorganization in widely separated cortical regions.

\section{MATERIALS AND METHODS}

The functional MRI and animal protocols (Logothetis et al., 1999) and data analysis methods (Teo et al., 1997; Wandell et al., 2000; Press et al., 2001; http://white.stanford.edu) have been described previously. The reader should consult those references for additional details.

MRI parameters. Multislice fMRI was performed by the use of multishot (segmented) gradient-recalled echo-planar imaging (EPI). Volumes of 13 or 17 slices of 1 or $2 \mathrm{~mm}$ were collected, each with a field of view of $128 \times 128 \mathrm{~mm}$ on a $256 \times 256$ matrix $(0.5 \times 0.5 \mathrm{~mm}$ in-plane resolution) and $2 \mathrm{~mm}$ slice thickness. The acquisition parameters were echo time, $20 \mathrm{msec}$; repetition time, $740 \mathrm{msec}$; flip angle, $50^{\circ}$; EPI zero phase, $8.192 \mathrm{msec}$ or $40 \%$ of phase steps; pulse length, $3.0 \mathrm{msec}$; spectral width, $100 \mathrm{kHz}$; line acquisition time, $1.28 \mathrm{msec}$; number of segments, 8 or 16; segment acquisition time (MRI readout window width), 20.48 msec, repetition time between slices, $37.59 \mathrm{msec}$; and number of excitations per phase encode step, one. To minimize the effects of inflow and of large drainage vessels, flip angles that were $10-20^{\circ}$ smaller than the computed Ernst angle were used.

Animal preparation. Three healthy juvenile monkeys weighing $6-9 \mathrm{~kg}$ were used for these experiments. All sessions were performed with great care to ensure the well-being of the monkeys, were approved by the local authorities (Regierungspraesidium), and were in full compliance with the guidelines of the European Community (EUVD 86/609/ EEC) for the care and use of laboratory animals. The monkeys were 


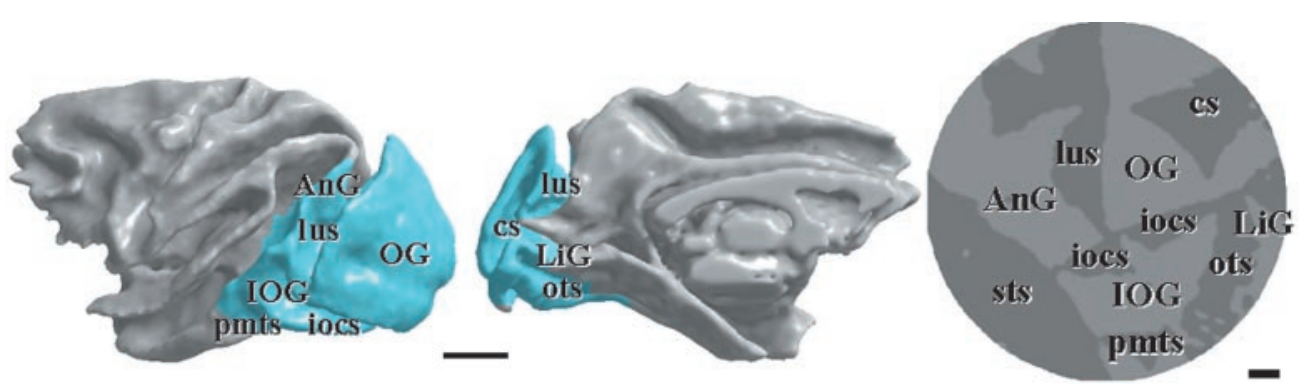

Figure 1. Segmentation and flattening of the brain. The three-dimensional renderings show the border between white matter and gray matter. The blue overlay depicts the region that is represented by the flattened image below. Several major sulci and gyri of the visual cortex are visible. In the flattened representation, these sulci and gyri are shown as dark and light bands, respectively. The positions of several major gyri and sulci are labeled: occipital gyrus $(O G)$, angular gyrus $(A n G)$, lingual gyrus $(L i G)$, lunate sulcus $(l u s)$, superior temporal sulcus (sts), calcarine (cs), inferior occipital gyrus $(I O G)$, occipitotemporal sulcus (ots), inferior occipital sulcus (iocs), and posterior middle temporal sulcus ( pmts). Scale bars: three-dimensional rendering, 10 mm; flattened representation, $5 \mathrm{~mm}$ (monkey B97).

anesthetized during the experiments. Details on the anesthesia protocol have been given previously (Logothetis et al., 2002). Briefly, the animals were preoxygenated, and anesthesia was induced with fentanyl $(31 \mu \mathrm{g} / \mathrm{kg})$, thiopental $(5 \mathrm{mg} / \mathrm{kg})$, and succinylcholine chloride $(3$ $\mathrm{mg} / \mathrm{kg})$. Muscle relaxation was achieved with mivacurium chloride (5 $\left.\mathrm{mg} \cdot \mathrm{kg}^{-1} \cdot \mathrm{h}^{-1}\right)$. Body temperature was kept constant, and lactated Ringer's solution was given at a rate of $10 \mathrm{mg} \cdot \mathrm{kg}^{-1} \cdot \mathrm{h}^{-1}$. Intravascular volume was maintained by administering colloids (hydroxyethyl starch, 30-50 $\mathrm{ml}$ over 1-2 $\mathrm{min}$ as needed). Monitoring within the magnet was mostly stable and reliable. The depth of anesthesia was assessed continuously by monitoring the vital signs of the monkey and responding accordingly, but because discomfort or stress would be masked by muscle paralysis, we first adjusted the depth of anesthesia before commencing paralysis, and we took the extra precaution of examining the concentration of plasma stress hormones (Logothetis et al., 1999). Anesthesia was always maintained at a level ( $0.4 \%$ isoflurane) that, combined with opiates, proved to ensure that the stress hormones remained within the physiological range.

Optical corrections. Two drops of $1 \%$ ophthalmic solution of the anticholinergic cyclopentolate hydrochloride were placed into each eye to achieve cycloplegia and mydriasis. Refractive errors were measured after the induction of paralysis, $\sim 1 \mathrm{hr}$ after the application of cyclopentolate. Contact lenses (Harte PMMA-Linsen Firma Wöhlk, Kiel, Germany) with the appropriate dioptric power were used to bring the animal's eye to a focus onto the stimulus plane (2 diopters).

Generation and positioning of the visual stimulus. Visual stimuli were created on a display using a resolution of $640 \times 480$ pixels with a $60 \mathrm{~Hz}$ frame rate. The display image was brought to the animal's eyes within the scanner by a fiber-optic projection system $(530 \times 400$ fibers $)$. The field of view was a $30^{\circ}$ horizontal $\times 23^{\circ}$ vertical visual angle; the focus was fixed at 2 diopters. Binocular presentations were accomplished through two independently positioned plastic fiber-optic glasses. A modified fundus camera (RC250; Zeiss, Thornwood, NY) served to position the stimulus, observe the eye fundus, and establish the $30^{\circ}$ horizontal $\times 23^{\circ}$ vertical calibration frame. This process ensured the alignment of the stimulus center with the fovea of each eye.

Stimuli. Visual field maps were measured using stimuli designed to produce a traveling wave of activity in retinotopically organized visual areas (Engel et al., 1994; Sereno et al., 1995; DeYoe et al., 1996). Expanding rings and rotating wedges were used to measure eccentric and angular maps, respectively. The stimuli were set to a period of $72 \mathrm{sec}$, and each scan included 14 total cycles. The first two were discarded to avoid transient effects, so that the data from each voxel spanned $864(72 \times 12)$ sec. At least three and usually four repeats of both the wedge and ring scans were performed on each animal.

The wedge and ring stimuli were made from various types of patterns. These included a contrast-reversing pattern on a uniform background, dot motion, or flickering color dots. These stimulus manipulations did not produce significant differences in the response; there was excellent consistency in the retinotopic maps obtained using these different stimuli. Hence, the visual field maps were constructed by combining data from these scans.

The traveling wave stimuli produce a temporal square wave of stimulation at each location in the visual field. The duty cycle of this square wave depends on the angular extent of the rotating wedge or the thick- ness of the expanding ring. In these experiments, a wedge of $90^{\circ}$ (duty cycle, $25 \%$ ) and a thin ring (duty cycle, $12.5 \%$ ) were used.

Response measurements. We describe only responses that are quite large, far above the statistical threshold. We summarize the strength of

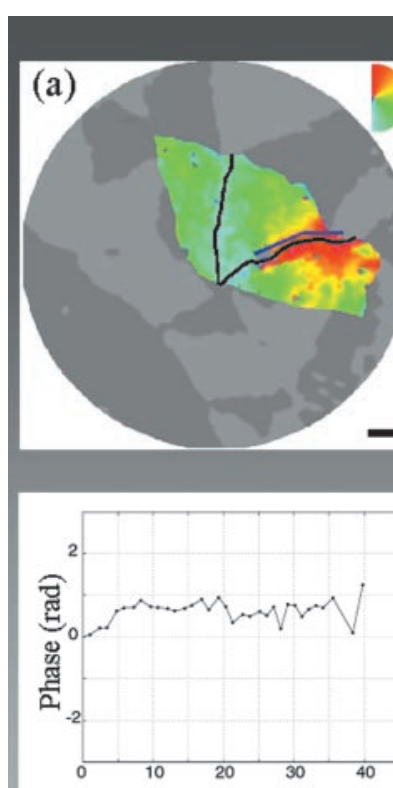

Pixel number along ROI
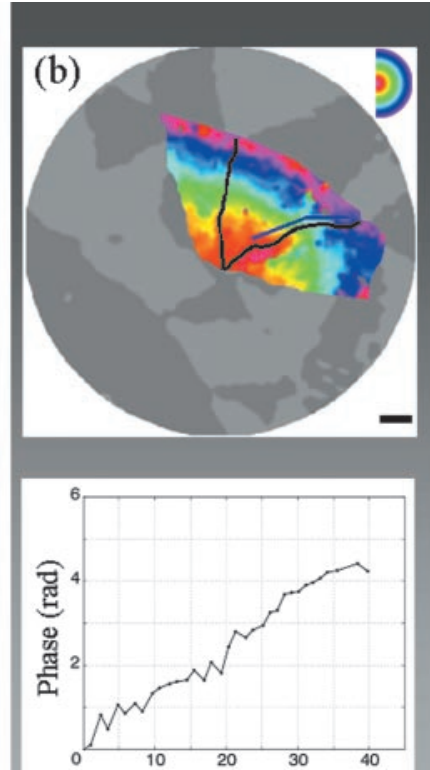

Pixel number along ROI

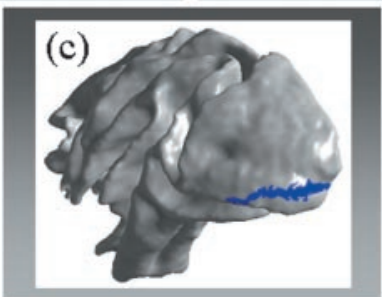

Figure 2. Methods to locate visual areas and analyze their topography. Templates that define the general topography of several visual areas were fit jointly to the rotating wedge $(a)$ and expanding ring $(b)$ phase data. Color overlays are shown at voxels within V1 and V2 with a coherence value of $>0.12$. The estimated boundary between areas V1 and V2 is shown by the black contour. The blue line represents an ROI within the template that traces a constant angular representation along the upper vertical meridian. The measured phase along this ROI is plotted below each of the flat maps for the rotating wedge and expanding ring data, respectively. The location of this ROI on the occipital gyrus is shown on the rendering in $c$. The details of the procedure are described in Materials and Methods. The maximal stimulus radius was $11^{\circ}$. Scale bar, $5 \mathrm{~mm}$. 
Figure 3. fMRI signal in a parasagittal slice measured in response to a rotating wedge stimulus. $a$, The coherence of the signal within all of the segmented cortical gray matter is shown. The green circles indicate regions of interest in $\mathrm{V} 1, \mathrm{~V} 2 \mathrm{~d}, \mathrm{~V} 3 \mathrm{v}$, and MT. $b$, Phase of the traveling wave caused by the rotating wedge stimulus. Several reversals in phase are visible (white arrows) and from these different visual areas can be identified. Color overlays are shown at voxels with a coherence value of $>0.20$. $c$, The amplitudes of the temporal harmonics of the fMRI signal are shown for each of the four ROIs in panel $a$. The $x$-axis measures the number of stimulus cycles per scan. The stimulus lasted 432 sec/cycle, and there were 12 cycles per scan; the scan duration was $5184 \mathrm{sec} / \mathrm{scan}$. The $x$-axis represents frequencies up to a maximum of four times the stimulus frequency (48 cycles per scan). The red lines are drawn leading to the fundamental stimulus frequency. The signals in $\mathrm{V} 1, \mathrm{~V} 2$, and MT are many SD above the noise level. The signals in V3 are detectable but weaker. Scale bar, $1 \mathrm{~cm}$ (monkey B97).
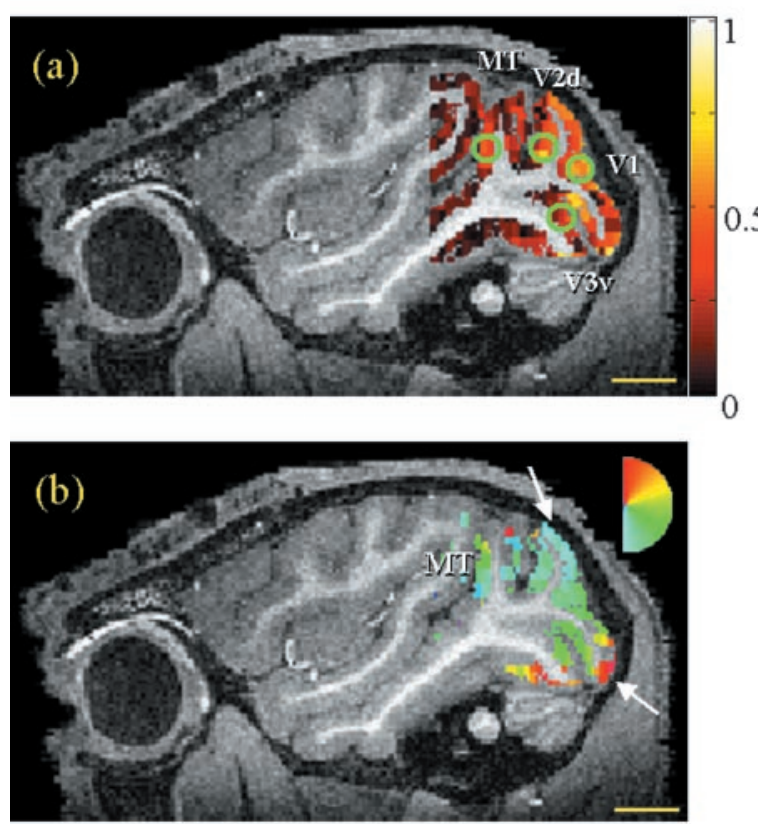

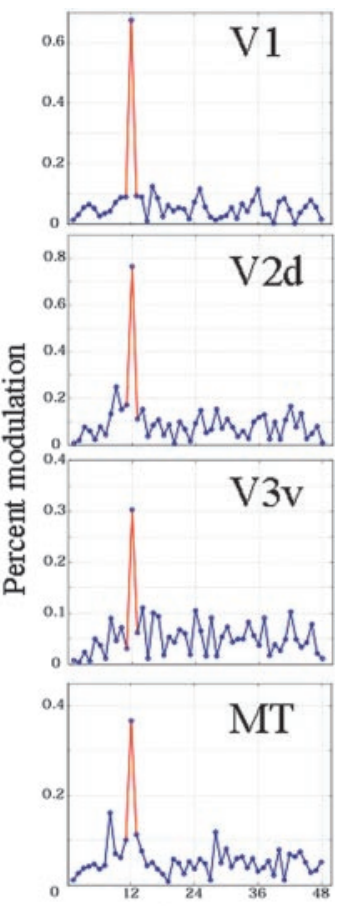

Cycles per scan these responses by measuring the coherence of the fMRI time series at the fundamental stimulus frequency. The coherence measures the ratio of the amplitude at the fundamental frequency to the signal variance, ranging between 0 and 1 . The formula for coherence is:

$$
\frac{A\left(f_{0}\right)}{\left(\sum_{\mathrm{f}=1}^{N / 2} A(f)^{2}\right)^{1 / 2}}
$$

The $A(f)$ terms represent the amplitude of the harmonic term at temporal frequency $f$, and the summation includes all the independent terms apart from the mean $(f=0)$. The traveling wave stimuli were presented at a fundamental frequency $f_{0}=1 / 72 \mathrm{~Hz}=0.0139 \mathrm{~Hz}$. At each voxel, the response phase at $f_{0}$ encodes either the preferred stimulus eccentricity (expanding ring) or the preferred stimulus angle (rotating wedge).

In the experiments described here, magnitude images were used. The fMRI signal was thus a real-valued function sampled at 144 points (volume acquisition time, $5.96 \mathrm{sec}$; sampling frequency, $0.167 \mathrm{~Hz}$ ), and its discrete Fourier transform included 72 independent amplitudes. If physiological noise were a white noise source, the amplitudes at all temporal frequencies would be approximately equal, and the expected coherence would be $1 / 72(0.014)$. In practice, however, the noise distribution is dominated by low-frequency terms. In brain regions that do not respond to the stimulus, the mean amplitude of the low-frequency physiological noise is $\sim 0.05$ with an SD of 0.02 . With this in mind, we consider the response of a voxel to be reliable when its amplitude is at least $3 \mathrm{SD}$ higher than the noise level (coherence $>0.11$ ); most of our analyses are restricted to voxels with a coherence of $>0.12$. In those instances in which we describe measurements combined from several voxels, the data are even further above the statistical threshold.

Data visualization and analysis. Gray matter was segmented and represented as topologically correct connected graphs using a T1-weighted anatomical scan (Teo et al., 1997). From this segmentation, we created three-dimensional renderings of the brain (Fig. 1, left) and flattened representations (Fig. 1, right). Light and dark shading on the flat map denote the gyri and sulci, respectively. The positions of the major landmarks in the occipital lobe in monkey B97 are indicated in Figure 1.

The positions of visual areas were defined by using measurements of the preferred angular and eccentric stimuli. Taken together, these measurements can define cortical regions that constitute a map of the visual field. In the case of areas V1-V3, where the basic organization is well established, we were able to use quantitative procedures. We created templates that define the expected visual topography in this group of visual areas. Using an interactive software tool, we indicated on a flat map the general region containing these areas. Furthermore, using a simple and rapid elastic deformation procedure (Fischer and Modersitzki, 1999), the template was deformed to minimize the sum of two terms: (1) the deviations between the template and the data and (2) the strain force of the elastic deformation. Once a minimum error solution was obtained, the boundary of the area was superimposed on the flat map.

Figure 2 shows an example of a fit to the area boundaries; the white contour indicates the estimated location of area V1 $\left(0-11^{\circ}\right)$. Furthermore, Figure 2 illustrates how we used the fitted template to derive a region of interest (ROI) that represents visual stimuli along a constant angle (isoangle). The blue line on the flat maps and the folded brain (Fig. $2 c$ ) shows an ROI that represents an isoangle path near the upper vertical meridian. Figure $2 a$ illustrates a flat map using a rotating wedge scan (top) and a plot of the measured phases along the fitted isoangle ROI (bottom). The phase values for this scan are nearly constant, as we expect for a rotating wedge scan. The corresponding data from an expanding ring experiment are shown in Figure $2 b$. These phase values increase along the ROI and measure the preferred stimulus eccentricity.

We also used this automated tool to measure visual field eccentricity (VFE) functions. We combined data from several isoangle regions within V1, V2, or V3 to derive the VFE functions. Specifically, each isoangle path was transformed back onto the folded brain, and distances were measured on this folded surface. All distances were specified with respect to the location that responded best to a stimulus at $10^{\circ}$ eccentricity; locations representing positions beyond $10^{\circ}$ eccentricity were assigned positive distances, and locations representing more foveal signals were assigned negative distances. Data from 4-10 isoangle paths were combined into a single VFE function, such as those shown in Figures 10-12.

Other visual areas, including V3A, V4, MT, and TEO, were estimated using graphical analyses of the traveling wave data. These are explained in graphs in Results.

\section{RESULTS}

We obtained measurements from three animals. The quality of the measurements differed somewhat from monkey to monkey. The clearest and most interpretable signals were obtained in animal B97. Results from the other two animals (E99 and H97) were mostly consistent, although there were some differences. Measurement artifacts can explain the main differences we observed among the animals. 

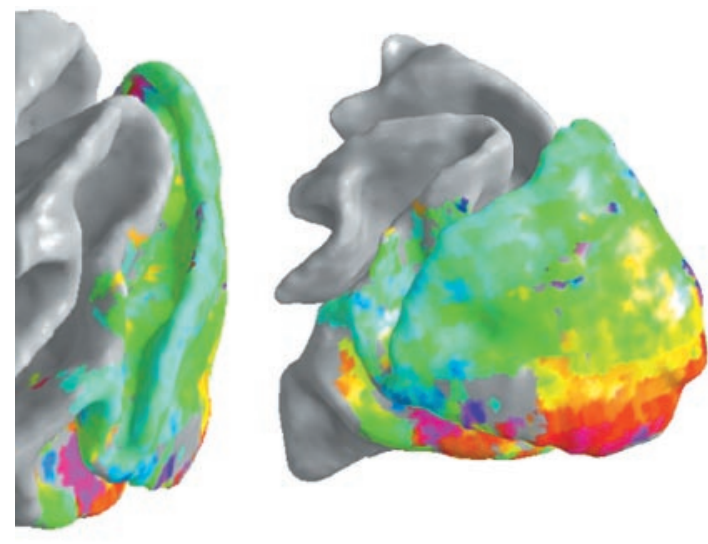

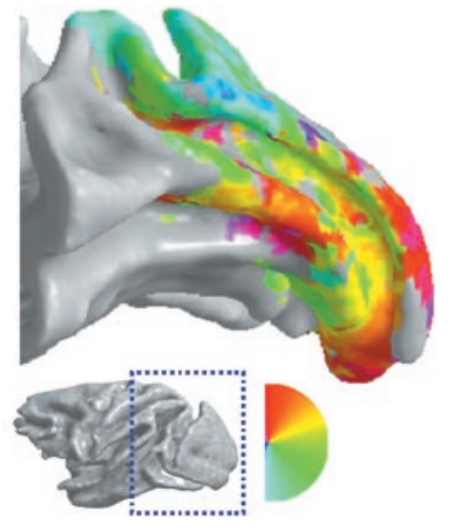

Figure 4. Traveling wave phase measurements from a rotating wedge. The region of the brain is indicated by the inset at the bottom right. The three images show the phase values on three views of the left hemisphere: lunate sulcus, operculum, and inferior occipital sulcus. Coherence, $>0.12$ (monkey B97). (a)

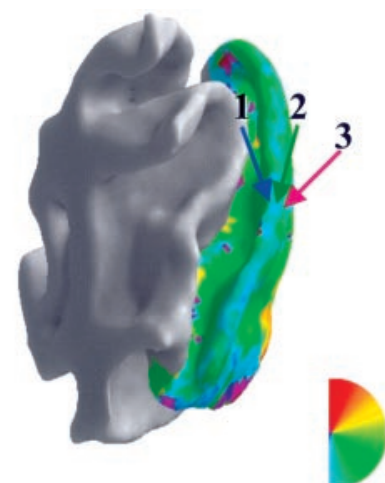

(c)

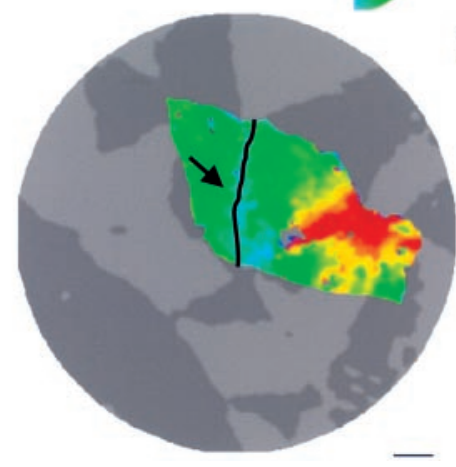

(b)

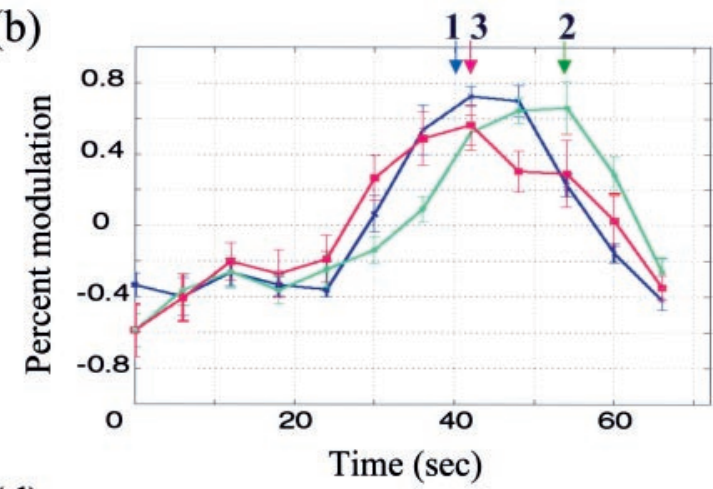

(d)

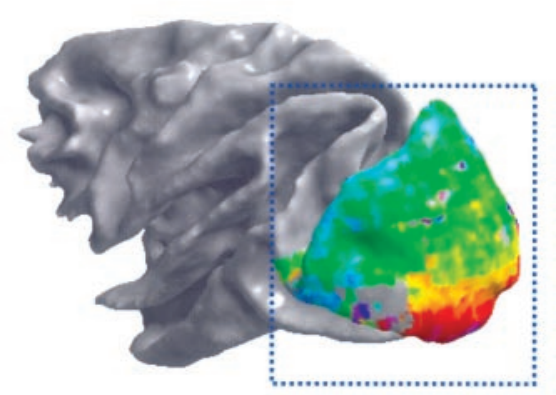

Figure 5. Signals near the dorsal boundary between V1 and V2. The color overlays in $a$, $c$, and $d$ measure the angular position that produces the strongest response (rotating wedge stimulus). $a$, Section of the posterior cortex indicating the locations of three ROIs near the peak of the occipital gyrus and descending into the lunate sulcus. $b$, Average time series at these locations. The phases of the time series of the blue (ROI 1) and magenta (ROI 3) locations are the same. ROI 2, indicated by the green arrow, has a time course with a significantly different phase, indicating a phase reversal as the measurements traverse the peak of the occipital gyrus. $c$, The same data are shown in a flattened representation along with the V1-V2 boundary derived from this reversal (black line). $d$, The data are also shown on the three-dimensional rendering at the bottom right. The dashed blue box indicates the approximate region of the flattened representation and the portion of cortex shown in a. Scale bar, $5 \mathrm{~mm}$; coherence, $>0.12$ (monkey B97).

\section{Spatial distribution of the traveling wave response}

The response coherence to a rotating wedge stimulus is very high near the occipital gyrus and in area MT/V5 (henceforth called MT). The coherence is smaller in more anterior portions of cortex. An average coherence map (four scans) in a typical parasagittal slice is shown in Figure $3 a$.

The borders between visual areas can be measured by the mirror reversals in the visual field map at horizontal and vertical meridian representations. Two such reversals are visible in the parasagittal view in Figure $3 b$ (arrows), defining the boundaries of the right hemifield representation on the operculum. Also, the angular retinotopic organization of area MT is evident on the posterior bank of the superior temporal sulcus (STS).

The response amplitudes at a range of temporal frequencies for ROIs located in areas V1-V3 and MT are plotted in Figure 3c. Each ROI (Fig. $3 a$, green circles) represents an $\sim 2 \mathrm{~mm}^{2}$ area of gray matter ( $\sim 1.7 \mathrm{~mm}$ thickness). These ROIs were chosen to fall at locations that represent the horizontal meridian. In this monkey, as well as the other two, the largest responses arise from areas V1, V2, and MT. Responses can be detected from areas that we have identified as V3, V3A, and TEO/V4A, but the responses in these areas are weaker. Responses from V4 are also present; these are weaker than responses from V1, V2, and MT but stronger than those from V3.

\section{Visual area identification}

\section{Angular representations}

Rotating wedge measurements from multiple imaging planes can be integrated into a single three-dimensional representation (Fig. 4). The three images show views of the lunate sulcus, operculum, and inferior occipital sulcus of the left hemisphere of one monkey. The color overlay represents the angular stimulus that evokes the largest response at each cortical location. A similar pattern of data was measured in two other animals. From the view of the operculum, the hemifield representation in V1 and the approximate positions of the V1 and V2 boundaries can be identified at the edges of the occipital gyrus: the dorsal peak near the lunate sulcus (lower vertical meridian, cyan) and the ventral peak near 
(a)

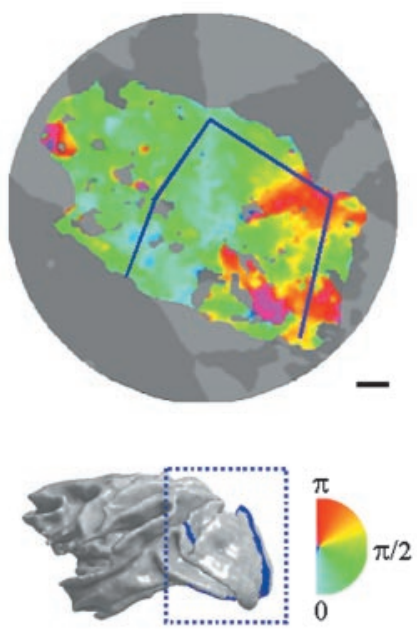

Figure 6. Visual area boundaries measured using reversals in the angular visual field maps. $a$, The color overlay represents the angular position that produces the strongest response (rotating wedge stimulus). Data from areas V1-V4 are shown on a flattened representation of the posterior cortex. The blue curve traces a path that traverses dorsal and ventral cortex, passing through the operculum. The preferred stimulus angle at locations along this curve is shown in $b$. The boundaries of several visual areas can be determined from the reversals (red arrows) in the curve representing the preferred stimulus angle. The inset shows the position of the blue curve on a three-dimensional rendering of the white-gray matter boundary. The dashed blue box shows the approximate region that was flattened for $a$. Scale bar, $5 \mathrm{~mm}$; coherence, $>0.12$ (monkey B97).

Figure 7. Traveling wave phase measurements from an expanding ring stimulus. The details are as in Figure 4, with the exception that the phase is derived from an expanding ring that measures eccentricity. The eccentricity map suggests a unified organization that spans most of the occipital lobe. (b)

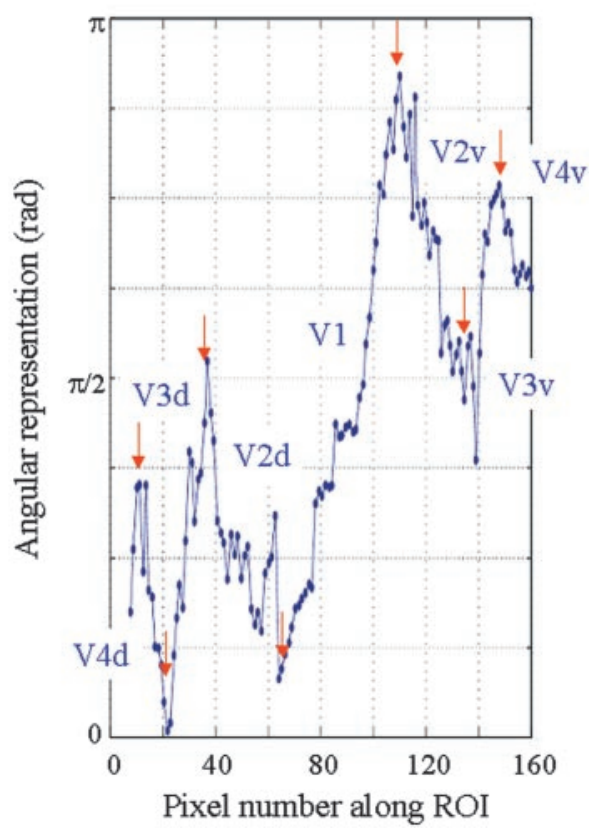

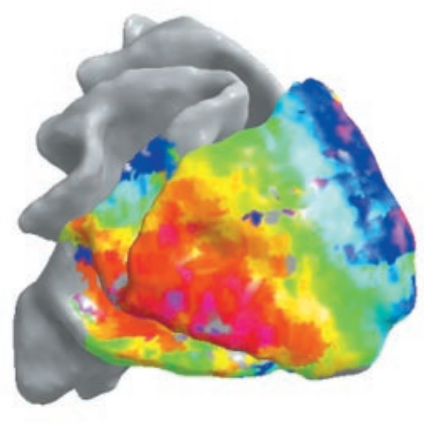

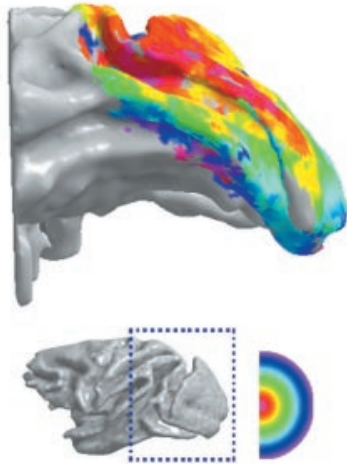

the inferior occipital sulcus (upper vertical meridian, red). There is a small portion of the operculum, seen in the middle image, where the signal coherence falls below 0.12 (gray); in this $3.5 \times$ $6.5 \mathrm{~mm}\left(23 \mathrm{~mm}^{2}\right)$ region, the signal level did not reach the required coherence threshold. From the expanding ring measurements (see below), we know that this region represents a part of the central visual field where it is difficult to obtain angular maps (Wandell, 1999; Tootell and Hadjikhani, 2001).

Quantitative measurements confirm that the phase of the traveling wave can be measured reliably. Figure $5 a$ shows an expanded view of the angular representation near the dorsal edge of the occipital gyrus. The arrows indicate three small ROIs (1 $\mathrm{mm}^{2}$ ) traversing the dorsal V1-V2 border. Figure $5 b$ shows the average time series measured at these three ROIs. The time series is the average of four separate scans ( 48 full rotations of the wedge stimulus). The ROIs at the end points $(1,3)$ are well apart from one another in the brain, but they have time series that are nearly identical in phase, indicating that they represent the same angle in the visual field. The time series from the middle ROI (2) differs substantially from the others (Watson-Williams $p<0.001$; Batschelet, 1981). These time series show the expected reversal in the visual field map as we traverse from V1 into V2, crossing the edge of the occipital gyrus and descending into the lunate sulcus. We have confirmed the presence of such reversals at many loca- tions along the black line on the flattened representation within Fig. $5 c$. On the basis of these reversals and the eccentricity maps shown below, we conclude that the black line represents the boundary between V1 and V2.

We have used this general approach to identify the locations of multiple visual areas, either by automated tools or by plotting comparable time series graphs. For example, the angular representation measured across an extensive region of striate and extrastriate cortex is shown in the flat map in Figure $6 a$. The color overlay again represents the angular stimulus that evokes the largest response at each cortical location. The superimposed curve in Figure $6 a$ shows a region of interest that traverses from dorsal to ventral cortex, passing through V1. The preferred stimulus angle is plotted as the blue points in Figure $6 b$. The phases measured in the dorsal regions span the lower quarter of the visual field ( $\pi / 2$ radians); area V1 spans a full hemifield ( $\pi$ radians); and the ventral visual areas span the upper quarter of the visual field ( $\pi / 2$ radians). The red arrows denote locations where the angular representation reverses along the selected ROI. We estimate the locations of the boundaries between the visual areas by combining many measured reversal points with information from eccentricity maps (described below).

We can identify two sources of noise in these measurements. First, at certain points along a boundary, the fMRI voxels span 
(a)

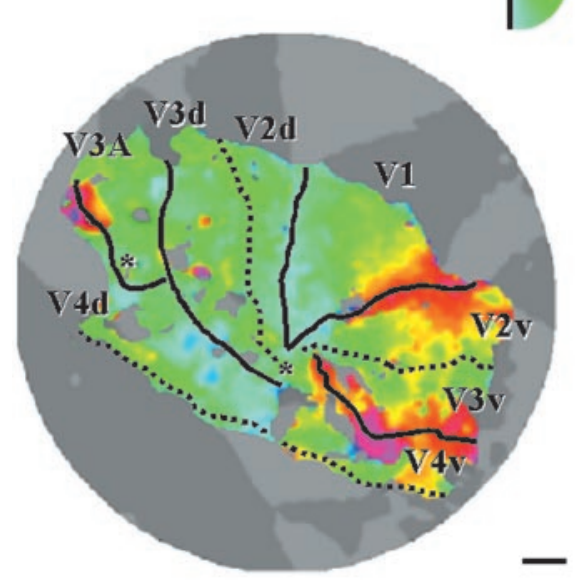

(b)

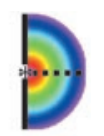

Figure 8. Visual area boundaries on the flattened representation. The color overlays indicate the angle $(a)$ and eccentricity $(b)$ of wedge and ring stimuli that elicit the maximal response. The estimated positions of visual areas V1-V3, $\mathrm{V} 3 \mathrm{~A}$, and V4 are also indicated. An asterisk is placed at cortical locations responding preferentially to near-foveal stimuli. Solid and dashed lines show vertical and horizontal meridia representations, respectively. Other details are as in Figure 5. two different visual areas; such averaging tends to reduce the phase measurements below their full extent. Second, the fMRI signals contain noise that can either increase or decrease the estimated range. The ability to identify any point along the boundary is limited by these noise contributions. The precision of the V1-V2 boundaries is on the order of 2-3 mm, based on repeated estimates using the automated boundary estimation tools described in Materials and Methods.

\section{Eccentricity representations}

The eccentricity representation can be measured using an expanding ring stimulus (Fig. 7). The data in this figure were obtained from the same animal as the data in Figures 4-6. They are shown in the same format, except that in Figure 7, the color overlay represents the stimulus eccentricity that evokes the largest response at each cortical location.

The images reveal a unified map of visual field eccentricity. Although the angular visual field representation divides the cortex into different areas, the eccentric field representation appears to unify the cortex into a single computational whole. It is apparent that the ventral surface is dominated by foveal signals. In human the foveal signals occupy a great deal of the ventral surface as well (Wade et al., 2002). The representation of eccentricity along the peak of the prelunate gyrus is distinctly more peripheral than the representation on the ventral surface. In human there is a corresponding segregation of peripheral and central representations between the dorsal and ventral visual cortex (Press et al., 2001).

We derived the locations of several retinotopic visual areas, shown in Figure 8, using a combination of automated tools and graphical analyses of the angular and eccentric measurements. To segment cortical regions into different areas we assume that (1) pieces of cortex that respond to the same portion of the visual field must belong to different visual areas, and (2) the angular and eccentricity representations must run in locally orthogonal directions. The visual area locations are outlined on the angular (Fig. $8 a$ ) and eccentric (Fig. 8b) measurements. As we discuss below, the properties of these areas identified using fMRI correspond well with the definitions derived from anatomy and electrophysiology.

The estimated locations of these visual areas (V1-V3, V3A, and V4) along with MT are shown on the parasagittal views in Figure 9. Each image shows a different animal. The automated segmentation tool described in Materials and Methods deter-
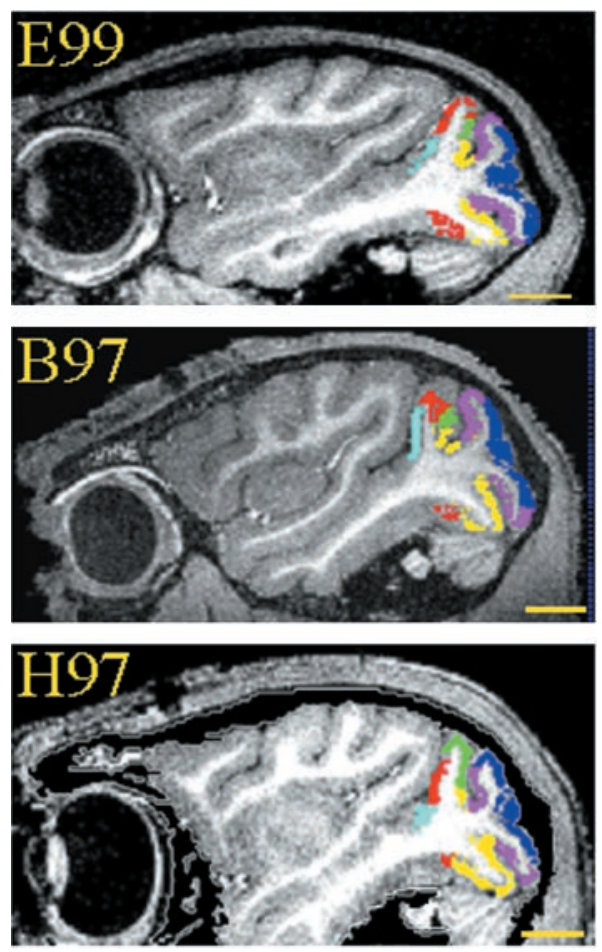

Figure 9. Estimated locations of the visual areas from three different monkeys (B97, E99, H97): V1 (blue), V2 (magenta), V3 (yellow), V3A (green), V4 (red), and MT (cyan). Scale bar, $1 \mathrm{~cm}$.

mined the locations of V1-V3. The positions of V3A, V4, MT, and the anterior border of V3d were located by inspection of the graphs, as described above. These data add to the evidence that the fMRI signal from traveling wave methods in the occipital cortex is colocalized with neural activity.

\section{Measurements of V1-V3}

Visual area sizes

Using our automated tools, we are able to quantify the dimensions of visual field representations within areas V1-V3. We estimate the width of area V1 spanning the upper to lower field representation at different eccentricities to be $30\left(11^{\circ}\right), 25\left(5^{\circ}\right)$, and $23\left(2.5^{\circ}\right) \mathrm{mm}$. Van Essen et al. (1984, their Fig. 4) reported 


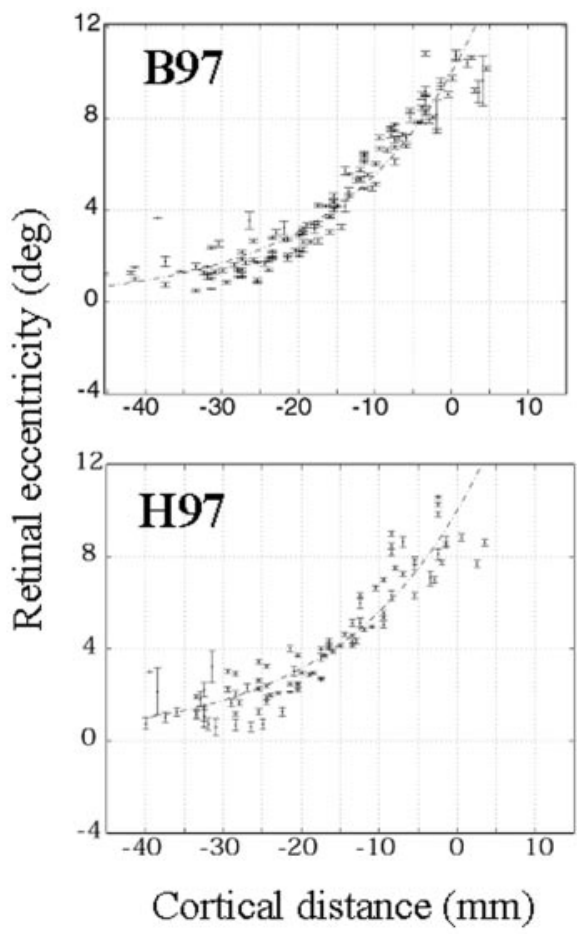

Figure 10. VFE function in V1 measured along the cortical surface. The $y$-axis measures visual field eccentricity (degrees), and the $x$-axis measures the distance along an isoangle ROI (distance) chosen using the automated procedure described in Figure 2. The distance along each ROI is measured relative to the $10^{\circ}$ eccentricity position. The smooth curve is the function $\operatorname{deg}=\exp (s \times$ distance $+\ln (10))$. Data from left and right hemispheres are combined in the plots. The two graphs show data from two monkeys (B97, H97).

corresponding distances from a juvenile macaque (1.5-3 kg): 27 , 25 , and $22 \mathrm{~mm}$. In our animals $(6-9 \mathrm{~kg})$, we estimate the distance from the central representation to the $11^{\circ}$ representation to be 35 $\mathrm{mm}$, whereas Van Essen et al. (1984) reported a distance of 27 $\mathrm{mm}$ for their smaller animals. The representation of the central $11^{\circ}$ spans an area of $\sim 945 \mathrm{~mm}^{2}$.

The combined V2 sections are approximately equal in size to V1. The V2 eccentricity direction spans $\sim 32 \mathrm{~mm}\left(11^{\circ}\right)$, and the angular dimension, combining V2d and V2v, equals $21 \mathrm{~mm}\left(5^{\circ}\right)$, a value very similar to that reported by Weller and Kaas (1983). Area V3, again combining V3d and V3v, is a bit smaller, spanning $20 \mathrm{~mm}$ in the eccentricity direction $\left(11^{\circ}\right)$ and $18 \mathrm{~mm}$ in the angular direction $\left(5^{\circ}\right)$. Hence, area V3 constitutes $\sim 40 \%$ of the area of V1 or V2. Gattass et al. (1988), measuring in macaque (3-4 kg), described the length of $\mathrm{V} 3$ from fovea to $11^{\circ}$ as $18 \mathrm{~mm}$ and the total width as $\sim 10 \mathrm{~mm}$. Hence, the measurement along the eccentricity dimension is in good agreement with ours, whereas their estimated width of V3 is smaller.

\section{Visual field eccentricity functions}

We measured the representation of the visual field eccentricity within areas V1-V3. The visual field eccentricity function we have used to describe human VFE functions is: $\operatorname{deg}=\exp (s \times$ distance $+\ln (10)$ ), where deg is visual field eccentricity; distance is measured from the point representing $10^{\circ}$ eccentricity (millimeters); and $s$ is a fitted scale factor (Baseler et al., 2002). In human V1, the scale factor, $s$, is consistently near 0.03-0.035.

The measurements from both hemispheres of area V1 in monkeys B97 and H97 are shown in Figure 10. In two animals (four

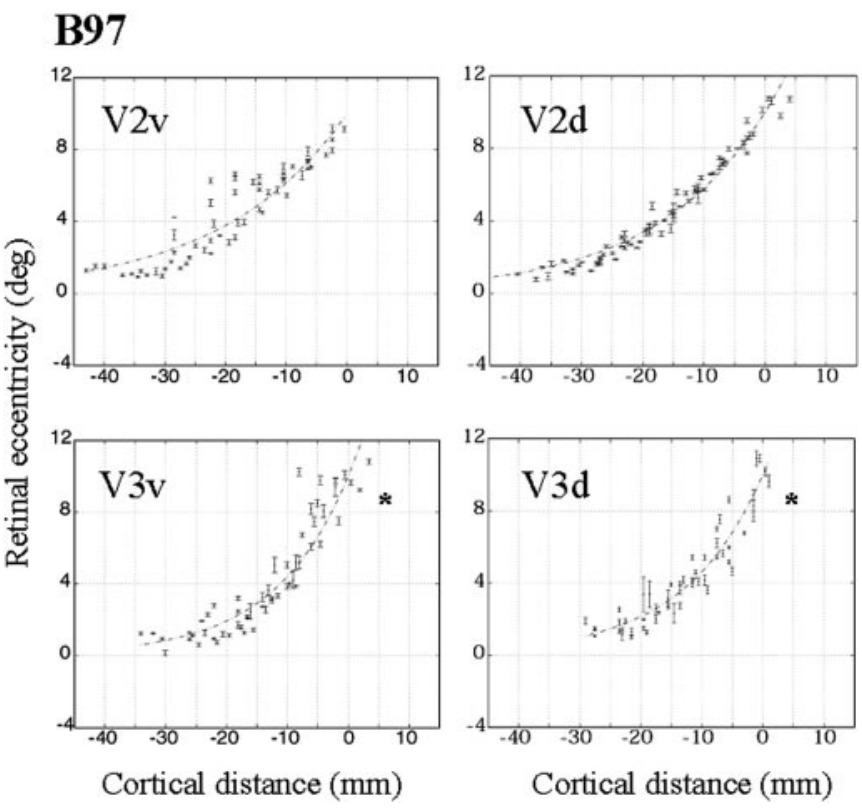

Figure 11. VFE functions in V2 and V3. Asterisks indicate the significantly increased scale factor of V3. Other details are as in Figure 10 (monkey B97).

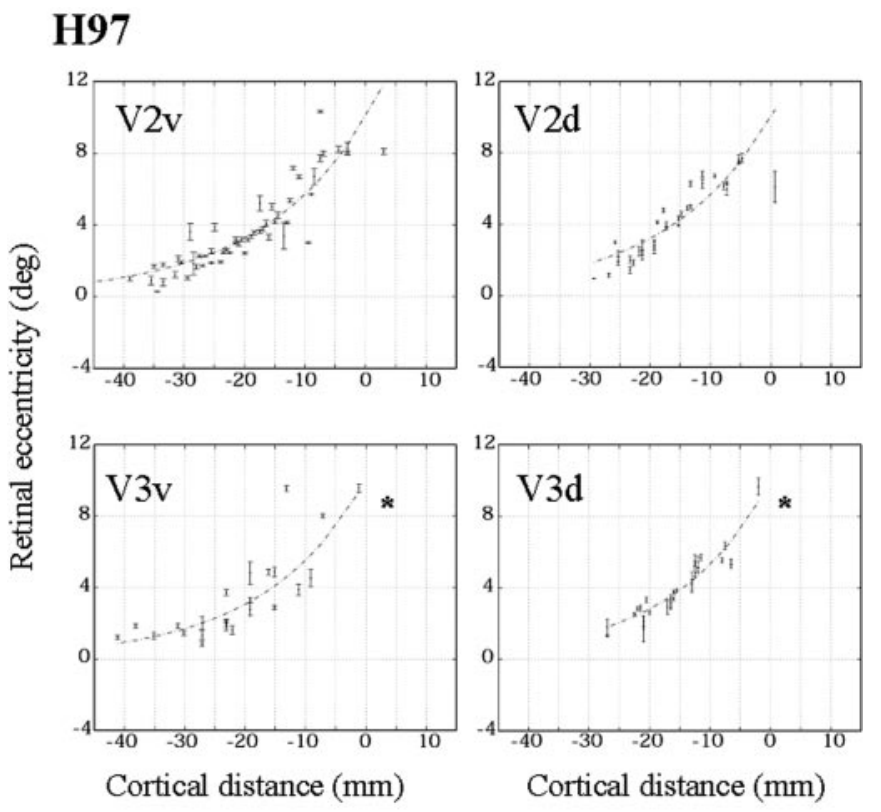

Figure 12. VFE functions in V2 and V3 of monkey H97. Other details are as in Figures 10 and 11.

hemispheres), the scale factor is consistently near 0.06, approximately twice the value in human. The increased scale factor means that the monkey represents the same visual eccentricity range in approximately half the cortical space. There was an anatomical artifact in the data from monkey E99 so that the entire function could not be measured. Estimates from the valid portions of the data were also consistent, with a scale factor near 0.06.

Figures 11 and 12 show the VFE plots along the length of areas $\mathrm{V} 2$ and V3. The curves from area V2 are quite similar to those in $\mathrm{V} 1$, with a scale factor of 0.06 , but the curve for V3 is significantly compressed (scale factor, 0.08). The compression is consistent 
(a)

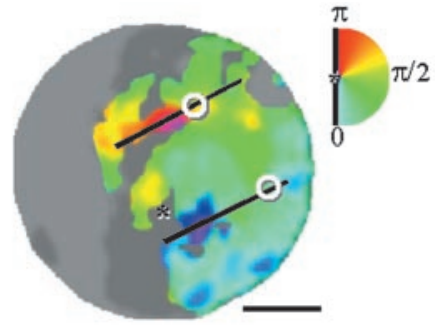

(c)

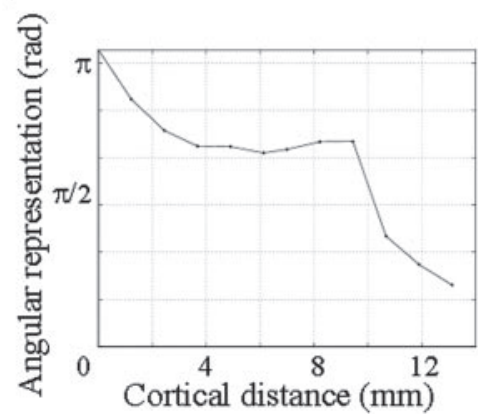

(b)

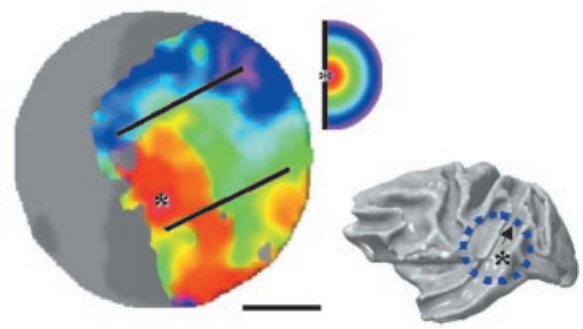

(d)

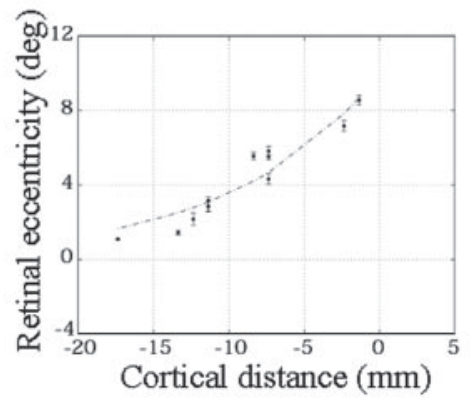

Figure 13. Visual field maps in the extrastriate cortex surrounding area MT. The location of the flattened region is indicated on the threedimensional inset at the top right. The arrow in the inset shows the direction of the eccentricity map running from the fovea (asterisk) along the STS. Expanded views of this section of the cortex are shown with color overlays that represent the preferred stimulus angle $(a)$ and preferred stimulus eccentricity $(b)$. Black lines indicate the estimated boundaries of MT out to $11^{\circ}$; asterisks locate the foveal representation. $c$, Preferred stimulus angle along an ROI drawn between the two white circles in $a$. The same expanded representation of the visual field just below the horizontal meridian is present at multiple eccentricities. $d$, VFE function for MT. Details are as in Figure 10. Scale bar, 5 $\mathrm{mm}$; coherence, $>0.12$ (monkey B97). with the visual topography of V3d measured by Gattass et al. (1988).

\section{Visual areas in anterior extrastriate cortex}

\section{Area V3A}

Van Essen and Zeki (1978) identified macaque V3A as a distinct, topographically organized visual area between areas V3 and V4 in the lunate and parieto-occipital sulci. Several portions of V3A can be distinguished from bordering areas V3 and V4 using myeloarchitectonic criteria (Gattass et al., 1988). Area V3A represents both superior and inferior visual quadrants in a single dorsal region. The region receives significant input from V3 as well as a direct projection from peripheral V1 (Zeki, 1980; Felleman et al., 1997).

The measured visual field maps anterior to V3 in all three animals have two main features that are consistent with these descriptions of V3A (Fig. 8). First, there is a full hemifield representation on the prelunate gyrus. In this representation, the lower visual field representation is adjacent to V3d, and part of the upper visual field representation is adjacent to V4d. Second, there is a well organized eccentricity map that runs perpendicular to the hemifield representation. The most central portion of the eccentricity representation responds best to signals near $5^{\circ}$ of eccentricity, not extending into central fovea. This peripheral bias is consistent with human eccentricity maps in a region that has been tentatively labeled V3A (Tootell et al., 1997; Press et al., 2001). The absence of a strong preference for central foveal signals may reflect differences in anatomical connectivity (Zeki, 1980), or they may simply reflect the relatively large receptive field sizes of V3A neurons (Van Essen and Zeki, 1978).

Although there is no clear demonstration of homology among macaque V3A, human V3A, and regions in corresponding positions in other primates (Krubitzer and Kaas, 1993; Kaas and Lyon, 2001), the visual field maps of V3A are generally consistent between human and macaque, as demonstrated here. However, the functional responsivities appear to differ (Tootell et al., 1997). Given the uncertainty about the relative contributions to the fMRI signal from spikes and graded input signals (Mathiesen et al., 1998; Logothetis et al., 2001), comparisons of functional responsivity using fMRI and electrophysiology should await further investigation.

\section{Area MT}

The traveling wave measurements show a retinotopic organization on the posterior bank of the STS in the location normally occupied by area MT (Fig. 13). The fMRI measurements define continuous angular (Fig. 13a) and eccentric (Fig. 13b) maps that can be seen on a flattened representation of a region ( $1 \mathrm{~cm}$ radius) centered on the putative location of MT. The short solid lines are drawn along the borders of a cortical region where the combined eccentric and angular representations define a hemifield representation. Given the cortical location and retinotopic organization, we conclude that this region is MT. Similar data were obtained from all three animals (six hemispheres).

There is a relatively expanded representation of the angular representation just below the horizontal midline (Fig. 13a,c). The expanded representation can be quantified by measuring the angular representation along a line drawn between the two white circles on the flat map. The graph in Figure $13 c$ shows that angles near the horizontal meridian span a relatively large cortical distance compared with other angles. Note the differences between this graph and the similar plot for V1, which has an equal distribution of angular representations (Fig. 2b). This irregular representation of the visual field in MT was first described using single-unit recording methods (Maunsell and Van Essen, 1987).

The representation of visual eccentricity in MT is compressed into a small size. Using automated methods, we estimate the VFE function scale factor to be 0.10 (Fig. 13d), significantly higher than the scale factor for V1 or V2 and slightly larger than the one measured for V3. The central $11^{\circ}$ of MT occupy a linear extent of $10-13 \mathrm{~mm}$ of cortex, and the angular dimension at various eccentricities $\left(2-10^{\circ}\right)$ occupies $10-13 \mathrm{~mm}$. For these animals, $\sim 6-9 \mathrm{~kg}$, the expected area for all of MT is $84-126 \mathrm{~mm}^{2}$ (Maunsell and Van Essen, 1987), whereas the fMRI estimates of the central $11^{\circ}$ are larger, $\sim 100-150 \mathrm{~mm}^{2}$. We find that for the representation of this part of the visual field, MT occupies $\sim 10-$ $15 \%$ of the area of V1. This estimate too is slightly higher than 
(a)

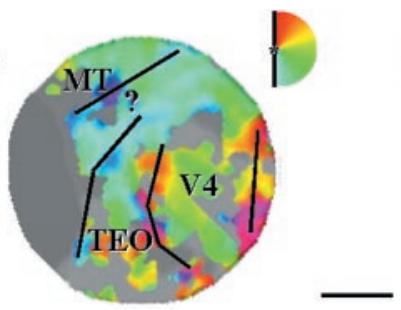

(b)

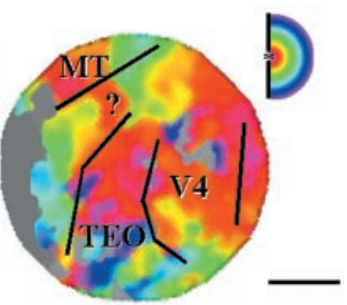

(c)

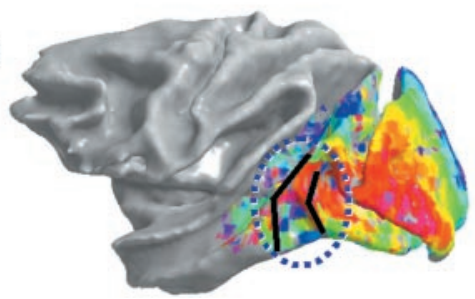

Figure 14. Visual field maps in the extrastriate cortex anterior to V4. The flat maps are views of the region near the putative location of TEO (1.5 $\mathrm{cm}$ radius). The color overlays represent the preferred stimulus angle (a) and preferred stimulus eccentricity $(b)$. Black lines indicate the estimated vertical meridia representations. The question mark indicates a lower-field representation between TEO and MT, sometimes labeled V4t. $c$, Three-dimensional representation of the gray-white matter boundary. The blue circle indicates the approximate region of the flattened representations, and black lines indicate the estimated position of TEO. Scale bar, $5 \mathrm{~mm}$; coherence, $>0.12$ (monkey B97).

the usual range of this ratio in the electrophysiological literature; for example, Weller and Kaas (1983) suggest 7\%.

\section{Area V4}

In all three animals, the angle and eccentricity maps contain a split hemifield representation beyond V3, organized concentrically around V1-V3. Figures 6 and 8 show this retinotopic organization adjacent to both dorsal and ventral V3 in a location usually identified as area V4. The fMRI signals from this region were relatively strong, exceeding the coherence level of 0.12 .

On the dorsal surface, the lower vertical meridian representa- tion in V4d borders V3d posteriorly and V3A anteriorly (Fig. 8a). The V4d quarter-field representation runs anterior to the horizontal meridian representation located near MT. On the ventral surface, the upper vertical meridian representation of $\mathrm{V} 4 \mathrm{v}$ shares a continuous border with $\mathrm{V} 3 \mathrm{v}$, whereas the horizontal meridian representation runs anterior to the upper vertical meridian representation of TEO (Fig. 14a).

The central visual field representation within V4 falls more on the ventral than the dorsal surface (Fig. 8b). This difference can be seen by comparing the large foveal representation in ventral V4 (red, inferior occipital sulcus) with the more peripheral signals in dorsal V4 (green, anterior lunate) in Figure 8b. A similar emphasis of the central visual field on the ventral surface has been observed in human (Wade et al., 2002).

\section{Area TEO}

A flat representation of angular and eccentric maps in the cortical region normally occupied by TEO is shown in Figure 14. The signals here were relatively weak, so that our measurements are tentative.

The angular and eccentricity maps approximate a hemifield representation whose borders are indicated in Figure 14, $a$ and $b$. The angular map contains a full hemifield, beginning with an upper-field representation (red) at the V4 boundary and continuing to a lower-field representation (cyan). Interestingly, this map runs in the same direction as the V4 map, without a mirror reversal between the two areas. Beyond this representation, there appears to be a further weak, retinotopic signal representing the lower visual field in the region sometimes described as $\mathrm{V} 4 \mathrm{t}$ (Desimone and Ungerleider, 1986) (Fig. 14a,b, question mark). The eccentricity map runs ventromedially from the foveal representation on the convexity of the inferior temporal gyrus. Because the central visual field occupies a large proportion of the cortical area, the eccentricity map is harder to define. The constant eccentricity representations in TEO extend those in V1, V2, V3v, and V4 (Fig. 14c), in agreement with single-unit measurements (Boussaoud et al., 1991). We observed a similar pattern in all three animals (six hemispheres).
Figure 15. Comparison of human $(a)$ and macaque $(b)$ eccentricity maps. The images on the left show the region of interest in the occipital lobe. The middle images show the eccentricity map out to $11^{\circ}$. The asterisk indicates the ventral foveal representation in the human brain; there is no precise correspondence to a distinct fovea in the macaque, although this foveal representation may correspond to the extended fovea in TEO. The locations of several visual area landmarks are labeled. The images on the right are overviews of the eccentric representation on two flat maps. Scale bar: human, $2 \mathrm{~cm}$; macaque, $1 \mathrm{~cm}$ (human $\mathrm{AB}$, monkey B97).
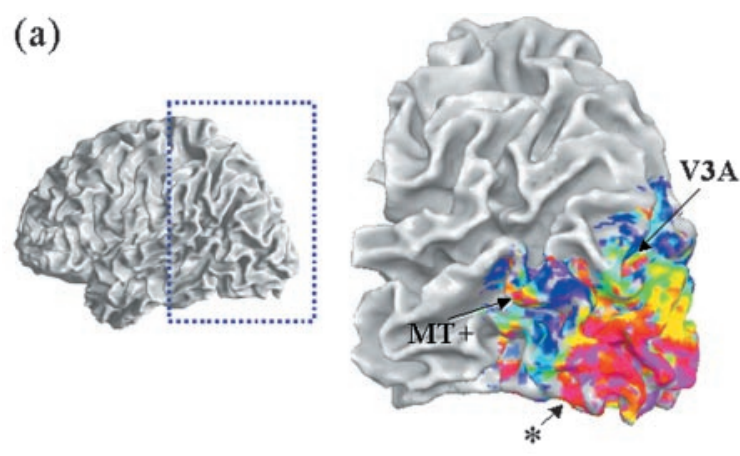

(b)

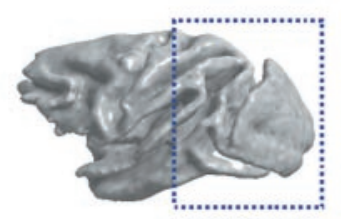

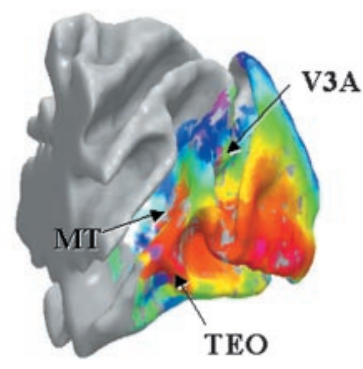
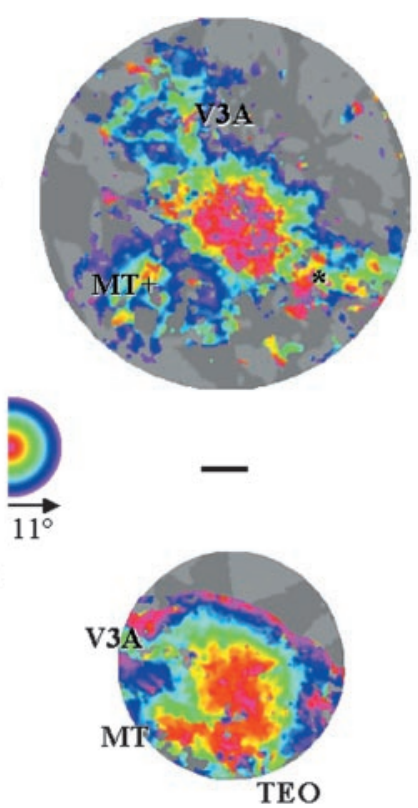


\section{DISCUSSION}

Establishing the location of visual areas using fMRI in the human brain has proved a useful tool for linking human visual pathway measurements to the wealth of data collected in monkey. There have been questions raised, however, about the reliability of the traveling wave methods and the quality of the fMRI localization with respect to neural signals. For example, there have been questions of whether the traveling wave methods could be relied on to reveal functional architecture (Zeki and Bartels, 1999; Bartels and Zeki, 2000) and whether there is a good concordance between neural maps and fMRI maps developed using a block design (Disbrow et al., 2000).

The quantitative agreement between the measurements described here and many of the topographic features in the visual cortex establish that (1) the traveling wave method provides an accurate view of the topography of visual cortex, and (2) using this design, the fMRI signals measure the local neural activity within this region of the visual cortex. The locations of major areas, including V1-V3, V3A, V4, TEO, and MT, and the distribution of the foveal and peripheral representations agree with well established anatomical landmarks and neuronal measurements. Hence, the high-field (4.7 T) magnet data described here support the hypothesis that traveling wave methods produce reliable visual field maps that colocalize with neural activity to within the resolution of these measurements. The spatial specificity observed in our experiments demonstrates once again the value of high-field imaging for studies relying on a high correlation between the spatial extent of the hemodynamic response and its underlying extent of neural activation.

The fMRI and traveling wave methods provide a broad view of the network of activities in the visual cortex. One useful application that takes advantage of this scale of measurement is the study of how the localized disruption of normal signals influences activity in the rest of the cortex. The reach of local biological disruptions can be quite far; for example, deletion of a single gene necessary for the cone transduction cascade leads to a substantial change in the maps in the visual cortex (Baseler et al., 2002). Another application is to use the high-resolution visual field maps for the study of ischemia or injury-induced cortical reorganization in the presence of different neurotrophic factors (e.g., brainderived neurotrophic factor). The methods developed here will help us visualize how relatively local events transform the responses in distant cortical regions.

An open question in computational neuroimaging is the homology between human and macaque visual areas (Wandell, 1999). The flat maps for eccentricity and angle obtained from monkey B97 and a human observer are compared in Figure 15. The angular and eccentricity maps in monkey and human brains are quite similar, apart from the overall scale. Common features include the large foveal representation at the confluence of the early visual areas, the concentric organization of the eccentricity map, the relative emphasis of foveal signals on the ventral (compared with dorsal) surface, and the distinct parafoveal band separating the foveal representations of V1-V3 from that of V3A. A notable difference is the presence of larger peripheral representations separating the early visual areas from the human MT+ complex and from the ventral foveal representation. The similarity of the fMRI maps between species provides a foundation for testing hypotheses about computational homologies based on stimulus-response measures.

\section{REFERENCES}

Baizer JS, Ungerleider LG, Desimone R (1991) Organization of visual inputs to the inferior temporal and posterior parietal cortex in macaques. J Neurosci 11:168-190.

Bartels A, Zeki S (2000) The architecture of the colour centre in the human visual brain: new results and a review. Eur J Neurosci 12:172-193.

Baseler HA, Brewer AA, Sharpe LT, Morland AB, Jägle H, Wandell BA (2002) Reorganization of human cortical maps caused by inherited photoreceptor abnormalities. Nat Neurosci 5:364-370.

Batschelet E (1981) Circular statistics in biology. New York: Academic.

Boussaoud D, Desimone R, Ungerleider LG (1991) Visual topography of area TEO in the macaque. J Comp Neurol 306:554-575.

Desimone R, Ungerleider LG (1986) Multiple visual areas in the caudal superior temporal sulcus of the macaque. J Comp Neurol 248:164-189.

DeYoe EA, Carman GJ, Bandettini P, Glickman S, Wieser J, Cox R, Miller D, Neitz J (1996) Mapping striate and extrastriate visual areas in human cerebral cortex. Proc Natl Acad Sci USA 93:2382-2386.

Disbrow EA, Slutsky DA, Roberts TP, Krubitzer LA (2000) Functional MRI at 1.5 tesla: a comparison of the blood oxygenation level-dependent signal and electrophysiology. Proc Natl Acad Sci USA 97:9718-9723.

Engel SA, Rumelhart DE, Wandell BA, Lee AT, Glover GH, Chichilnisky EJ, Shadlen MN (1994) fMRI of human visual cortex [letter]. Nature [Erratum (1994) 370:106] 369:525.

Engel SA, Glover GH, Wandell BA (1997) Retinotopic organization in human visual cortex and the spatial precision of functional MRI. Cereb Cortex 7:181-192.

Felleman DJ, Burkhalter A, Van Essen DC (1997) Cortical connections of areas V3 and VP of macaque monkey extrastriate visual cortex. J Comp Neurol 379:21-47.

Fischer B, Modersitzki J (1999) Fast inversion of matrices arising in image processing. Numerical Algorithms 22:1-11.

Gattass R, Sousa AP, Gross CG (1988) Visuotopic organization and extent of V3 and V4 of the macaque. J Neurosci 8:1831-1845.

Kaas JH, Lyon DC (2001) Visual cortex organization in primates: theories of V3 and adjoining visual areas. Prog Brain Res 134:285-295.

Krubitzer LA, Kaas JH (1993) The dorsomedial visual area of owl monkeys: connections, myeloarchitecture, and homologies in other primates. J Comp Neurol 334:497-528.

Logothetis NK, Guggenberger H, Peled S, Pauls J (1999) Functional imaging of the monkey brain. Nat Neurosci 2:555-562.

Logothetis NK, Pauls J, Augath M, Trinath T, Oeltermann A (2001) Neurophysiological investigation of the basis of the fMRI signal. Nature 412:150-157.

Logothetis NK, Merkle H, Augath M, Trinath T, Ugurbil K (2002) Ultra-high resolution fMRI in monkeys with implanted RF coils. Neuron 35:227-242.

Mathiesen C, Caesar K, Akgoren N, Lauritzen M (1998) Modification of activity-dependent increases of cerebral blood flow by excitatory synaptic activity and spikes in rat cerebellar cortex. J Physiol (Lond) 512:555-566.

Maunsell JH, Van Essen DC (1987) Topographic organization of the middle temporal visual area in the macaque monkey: representational biases and the relationship to callosal connections and myeloarchitectonic boundaries. J Comp Neurol 266:535-555.

Morel A, Bullier J (1990) Anatomical segregation of two cortical visual pathways in the macaque monkey. Vis Neurosci 4:555-578.

Press WT, Brewer AA, Dougherty RF, Wade AR, Wandell BA (2001) Visual areas and spatial summation in human visual cortex. Vision Res 41:1321-1332.

Sereno ME, Trinath T, Augath M, Logothetis NK (2002) Threedimensional shape representation in monkey cortex. Neuron 33:635-652.

Sereno MI, Dale AM, Reppas JB, Kwong KK, Belliveau JW, Brady TJ, Rosen BR, Tootell RB (1995) Borders of multiple human visual areas in humans revealed by functional MRI. Science 268:889-893.

Teo PC, Sapiro G, Wandell BA (1997) Creating connected representations of cortical gray matter for functional MRI visualization. IEEE Trans Med Imaging 16:852-863.

Tolias AS, Smirnakis SM, Augath MA, Trinath T, Logothetis NK (2001) Motion processing in the macaque: revisited with functional magnetic resonance imaging. J Neurosci Methods 21:8594-8601.

Tootell RB, Mendola JD, Hadjikhani NK, Ledden PJ, Liu AK, Reppas JB, Sereno MI, Dale AM (1997) Functional analysis of V3A and related areas in human visual cortex. J Neurosci 17:7060-7078.

Tootell RB, Hadjikhani N (2001) Where is dorsal V4' in human visual cortex? Retinotopic, topographic and functional evidence. Cereb Cortex 11:298-311.

Vanduffel W, Fize D, Mandeville JB, Nelissen K, Van Hecke P, Rosen BR, Tootell RB, Orban GA (2001) Visual motion processing investi- 
gated using contrast agent-enhanced fMRI in awake behaving monkeys. Neuron 32:565-577.

Van Essen DC, Zeki SM (1978) The topographic organization of rhesus monkey prestriate cortex. J Physiol (Lond) 277:193-226.

Van Essen DC, Newsome WT, Maunsell JH (1984) The visual field representation in striate cortex of the macaque monkey: asymmetries, anisotropies, and individual variability. Vision Res 24:429-448.

Van Essen DC, Lewis JW, Drury HA, Hadjikhani N, Tootell RB, Bakircioglu M, Miller MI (2001) Mapping visual cortex in monkeys and humans using surface-based atlases. Vision Res 41:1359-1378.

Wade AR, Brewer AA, Rieger JW, Wandell BA (2002) Functional measurements of human ventral occipital cortex: retinotopy and color. Philos Trans R Soc Lond B Biol Sci 357:963-973.
Wandell BA (1999) Computational neuroimaging of human visual cortex. Annu Rev Neurosci 22:145-173.

Wandell BA, Chial S, Backus B (2000) Visualization and Measurement of the Cortical Surface. J Cognit Neurosci 12:739-752.

Weller RE, Kaas JH (1983) Retinotopic patterns of connections of area 17 with visual areas V-II and MT in macaque monkeys. J Comp Neurol 220:253-279.

Zeki S (1980) A direct projection from area V1 to area V3A of rhesus monkey visual cortex. Proc R Soc Lond B Biol Sci 207:499-506.

Zeki S, Bartels A (1999) The clinical and functional measurement of cortical (in)activity in the visual brain, with special reference to the two subdivisions (V4 and V4 alpha) of the human colour centre. Philos Trans R Soc Lond B Biol Sci 354:1371-1382. 